\title{
Linking supernovae and supernova remnants. Time-dependent injection in SN1987A and gamma-ray spectrum of IC443
}

\author{
O. Petruk ${ }^{1,2}$, S. Orlando ${ }^{1}$ and M. Miceli ${ }^{1,3}$ \\ ${ }^{1}$ INAF-Osservatorio Astronomico di Palermo, Piazza del Parlamento 1, 90134 Palermo, Italy \\ ${ }^{2}$ Inst. Appl. Probl. in Mechanics and Mathematics, Naukova Street, 3-b Lviv 79060, Ukraine \\ ${ }^{3}$ Dip. di Fisica e Chimica, Univ. di Palermo, Piazza del Parlamento 1, 90134 Palermo, Italy
}

\begin{abstract}
Acceleration times of particles responsible for the gamma-rays in supernova remnants (SNRs) are comparable with SNR age. If the number of particles starting acceleration was varying during early times after the supernova explosion then this variation should be reflected in the shape of the gamma-ray spectrum. In order to analyse this effect, we consider the time variation of the radio spectral index in SN1987A and solution of the non-stationary equation for particle acceleration. We reconstruct evolution of the particle injection in SN1987A, apply it to derive the particle momentum distribution in IC443 and model its gamma-ray spectrum. We show that: i) observed break in the proton spectrum around $50 \mathrm{GeV}$ in IC443 is a consequence of the variation of the cosmic ray injection; ii) shape of the hadronic gamma-ray spectrum in SNRs critically depends on the temporal variation of the cosmic ray injection in the immediate post explosion phases.
\end{abstract}

Keywords. (stars:) supernovae: individual (SN1987A), (ISM:) supernova remnants: individual (IC443), acceleration of particles, gamma-rays

\section{Introduction}

The steady-state particle injection and the stationary acceleration are typically adopted in interpretation of the $\gamma$-ray spectra from supernova remnants (SNRs). Are these regimes justified to simulate the momentum distributions of the highest energy particles? Is it correct to consider the stationary solution for diffusive shock acceleration if injection is not constant or not impulsive? What consequences could be if the number of particles starting acceleration in the aftermath of the supernova event varies? May this early evolution be reflected after some time in the spectrum of the most energetic emission? In order to answer these questions, let us consider the general time-dependent equation.

\section{Time-dependent particle acceleration at the SNR shock}

The distribution function $f(t, x, p)$ for cosmic rays which accelerate diffusively on the shock of SNRs is a solution of the non-stationary equation (e.g., Skilling 1975, Jones 1990):

$$
\frac{\partial f}{\partial t}+u \frac{\partial f}{\partial x}=\frac{\partial}{\partial x}\left[D \frac{\partial f}{\partial x}\right]+\frac{1}{3} \frac{d u}{d x} p \frac{\partial f}{\partial p}+Q
$$

where $t$ is the time, $x$ the spatial coordinate, $p$ the momentum, $D$ the diffusion coefficient, $u$ the flow velocity in the shock reference frame, $Q$ the injection term. We assume that 


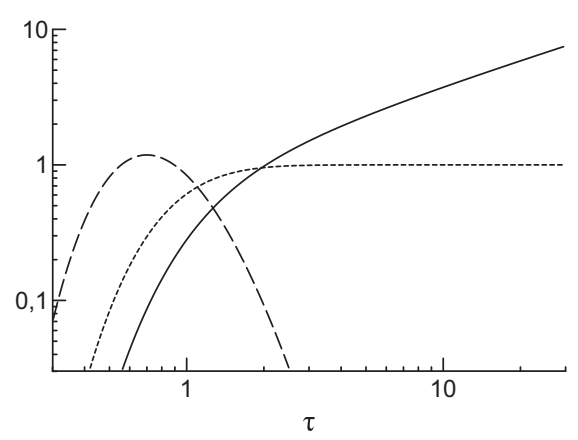

Figure 1. The distribution function $\varphi_{\mathrm{o}}$ (dashed line) and the integral in the solution (2.3) for $Q_{\mathrm{t}}=1$ (dotted line) and $Q_{\mathrm{t}}=\tau^{0.7}$ (solid line) versus the dimensionless time $\tau$.

particles are injected at the shock $(x=0)$ with the momentum $p_{\mathrm{i}}$ :

$$
Q(t, x, p)=\frac{\eta n_{1} u_{1}}{4 \pi p_{\mathrm{i}}^{2}} \delta\left(p-p_{\mathrm{i}}\right) \delta(x) Q_{\mathrm{t}}(t),
$$

where the parameter $\eta$ is the injection efficiency (the density fraction of accelerated particles); it is defined to be the value of the efficiency at the late steady-state regime. The term $Q_{\mathrm{t}}(t)$ represents the time evolution of the injection; obviously, it is unity in the steady-state regime. The indices '1', '2' and 'o' correspond to upstream, downstream and to the shock position.

The test-particle solution of equation (2.1) for the steady-state injection $Q_{\mathrm{t}}=1$ was derived by Drury (1983), Forman \& Drury (1983). The solution for the time-dependent injection was derived by Petruk \& Kopytko (2016):

$$
f_{\mathrm{o}}(t, p)=\mathrm{f}_{\mathrm{o}}(p) \int_{0}^{\tau} Q_{\mathrm{t}}\left(\tau-\tau^{\prime}\right) \varphi_{\mathrm{o}}\left(\tau^{\prime}\right) d \tau^{\prime},
$$

where

$$
\mathrm{f}_{\mathrm{o}}(p)=\frac{\eta n_{1}}{4 \pi p_{\mathrm{i}}^{3}} \frac{3 \sigma}{\sigma-1}\left(\frac{p}{p_{\mathrm{i}}}\right)^{-s_{\mathrm{f}}}
$$

is the solution of the stationary equation with the spectral index $s_{\mathrm{f}}=3 \sigma /(\sigma-1), \sigma=$ $u_{1} / u_{2}$ is the shock compression factor and the probability distribution is (Forman \& Drury (1983), Petruk \& Kopytko (2016))

$$
\varphi_{\mathrm{o}}(\tau)=\frac{e^{2 A}}{2^{2 A+1} \sqrt{\pi}} \frac{e^{-\xi(\tau)^{2}}}{\tau^{A / 2+1}}\left(\mathrm{H}_{A+1}(\xi)-2 \tau^{1 / 2} \mathrm{H}_{A}(\xi)\right),
$$

where $\mathrm{H}_{m}(x)$ is the Hermite polynomial, $\tau=t / t_{1}, t_{1}=4 D_{1} / u_{1}^{2}, \xi(\tau)=\tau^{1 / 2}+A /\left(2 \tau^{1 / 2}\right)$, $A=s_{\mathrm{f}} / \alpha, \alpha$ the index in the diffusion coefficient $D(p) \propto p^{\alpha}$.

It is clear from (2.3) and Fig. 1 that the widely-used stationary solution for the particle acceleration may actually be considered only in the case when $Q_{\mathrm{t}}=1$ and values $\tau \gg \tau_{*}$ where $\tau_{*}$ marks the maximum in the distribution $\varphi_{\mathrm{o}}(\tau)$. Note that $\tau=t / t_{1}(p)$ depends on two variables, $t$ and $p$. Therefore, the probability $\varphi_{\mathrm{o}}$ is a two-dimensional distribution.

In order to relate the unitless time $\tau$ to the physical time $t$ and momentum $p$, the following expressions have to be used: $t=\tau t_{\text {age }}$ and $p=\tau^{-1 / \alpha} p_{\max }$ where $t_{\text {age }}$ is the SNR age and $p_{\max }$ is the maximum particle momentum. Therefore, the larger $\tau$ corresponds to the larger $t$ and the smaller $p$. 

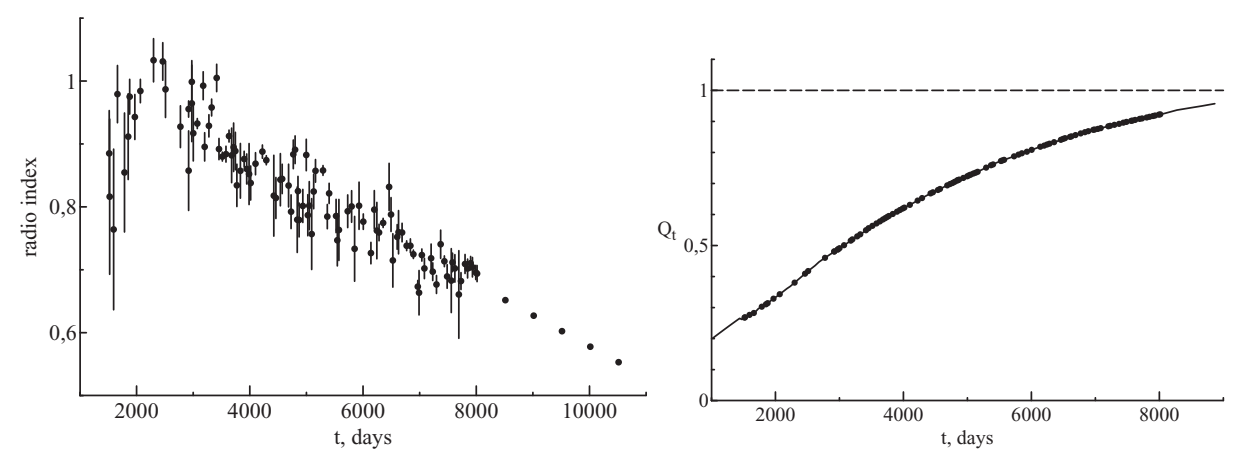

Figure 2. Observed evolution of the radio index in SN1987A (on the left) from Zanardo et al. (2013) and the temporal dependence of the particle injection derived from these data (on the right; dots corresponds to the period of the radio observations; dashed line represents the case of the steady-state injection). Adapted from Petruk et al. (2017).

It is important that the highest-energy particles has $\tau \ll 1$ where the integral in the solution is considerably less than unity. This means that the highest-energy particles (which actually are responsible for the $\gamma$-rays) cannot be described by the stationary solution.

Instead, the radio-emitting electrons has $\tau \gg \tau_{*}$ and may be described by the stationary solution if the injection is characterized by $Q_{\mathrm{t}}=1$ (dotted line). However, if $Q_{\mathrm{t}} \neq 1$ then also the radio emission is affected by the temporal evolution of injection. It may be noted from Fig. 1 (solid line) that the injection term of the form $Q_{\mathrm{t}} \propto \tau^{\beta}$ affects, at large $\tau$, the spectral index of the accelerated particle distribution. The solution in this case may be written in the form (Petruk \& Kopytko (2016))

$$
f_{\mathrm{o}}(t, p) \propto p^{-\left(s_{\mathrm{f}}+\alpha \beta\right)} .
$$

The radio spectral index $\alpha_{\mathrm{r}}$ is known to be related to the index of the particle distribution. With that relation, we come to the simple formula

$$
\alpha_{\mathrm{r}}=\frac{s_{\mathrm{f}}+\alpha \beta-3}{2}
$$

which directly connects the spectral index and $\beta$, with typical values of the adiabatic constant $\gamma=5 / 3$ (therefore $\sigma=4$ and $s_{\mathrm{f}}=4$ ) and $\alpha=1$ for the Bohm-like diffusion.

\section{Radio index of SN1987A and evolution of particle injection}

The formula (2.7) allows us to reconstruct the temporal evolution of the particle injection in SN1987A (see Petruk et al. (2017) for more details). We use the detailed observations of the radio index in SN1987A (Fig. 2 left) given by Zanardo et al. (2013). From these data we obtain a series of $\beta$, one for each observed $\alpha_{\mathrm{r}}$, and then represent the evolution of injection as a piecewise step function. This function is shown on Fig. 2 right and is represented by the following expression

$$
Q_{\mathrm{t}}(t)=q_{1} t^{\beta_{\mathrm{i}}} \prod_{m=2}^{i} t_{\mathrm{m}}^{\beta_{\mathrm{m}-1}-\beta_{\mathrm{m}}}, \quad t_{\mathrm{i}} \leqslant t \leqslant t_{\mathrm{i}+1} .
$$

It is derived from the condition that the function $Q_{\mathrm{i}}=q_{\mathrm{i}} t_{\mathrm{i}}^{\beta_{\mathrm{i}}}$ in a single step has to connect the two nearby points $\left(t_{\mathrm{i}}\right.$ and $\left.t_{\mathrm{i}+1}\right)$ and the normalization $q_{\mathrm{i}+1}$ of each step is fixed by the function on the previous one: $q_{\mathrm{i}} t_{\mathrm{i}+1}^{\beta_{\mathrm{i}}}=q_{\mathrm{i}+1} t_{\mathrm{i}+1}^{\beta_{\mathrm{i}+1}}$. The value of $q_{\mathrm{o}}$ is given by requirement to provide $Q_{\mathrm{t}}=1$ at large $t$. 


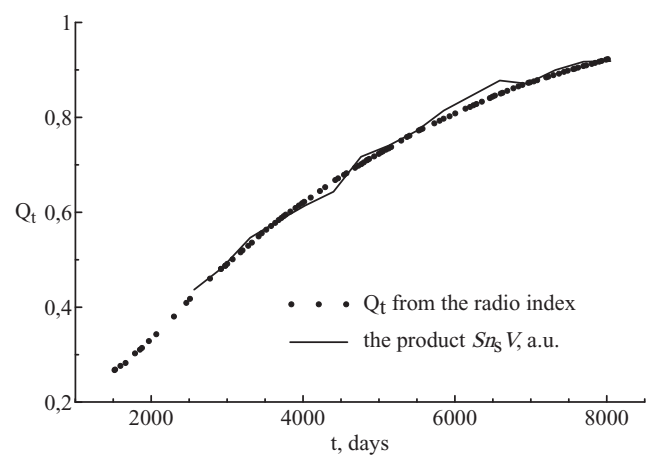

Figure 3. Evolution of $Q_{\mathrm{t}}$ during the period of the radio observations (dots) and of the product $S n_{\mathrm{s}} V$ in 3-D model of SN1987A by Orlando et al. (2015) (line, in arbitrary units).

The observations are available from 1517 to 8014 days. After the last observed point, we extrapolate (Fig. 2 left, dots without errors) the behavior of the radio index using the fit suggested by Zanardo et al. (2013), up to the value $\alpha_{\mathrm{r}}=0.5$ and then keep it constant. Before the day 1517, we assume rather arbitrary $Q_{\mathrm{t}}$ to be constant till the day 100 and then $Q_{\mathrm{t}}(t) \propto t^{0.8}$ after this day. Such a choice provides us with a good fit for the highest-energy part of the $\gamma$-ray spectrum of IC443 considered in the next section.

Right plot on Fig. 2 demonstrates clearly that the injection efficiency cannot be considered constant until some age of the remnant of supernova, when the radio index reaches a saturated level close to 0.5. This observational evidence needs to be understood from a theoretical point of view. Different factors is expected to influence the injection (e.g. shock strength, level of turbulence). In order to make initial insight, let us assume that the injected particles are a constant fraction $\eta$ of the flux incoming through the shock. Then $Q_{\mathrm{t}}$ has to be proportional to the product of the shock surface $S$, post-shock density $n_{\mathrm{s}}$ and shock velocity $V$. In order to check this, we consider the three-dimensional model of this SNR by Orlando et al. (2015). Fig. 3 shows the product $S n_{\mathrm{S}} V$ in comparison with the time dependence of $Q_{\mathrm{t}}$. The model shows that, shortly after the day 2000, the forward shock enters the HiI region with a constant density and freely expands there with roughly constant velocity. Therefore, the prominent change in the injection efficiency happens, to the large extent, because the surface of the shock from explosion increases considerably during the immediate aftermath of supernova (at later stages, the shock radius increases much slowly).

\section{Non-stationary gamma-ray spectrum of IC443}

We substitute the function $Q_{\mathrm{t}}(t)$ derived above into the non-stationary solution (2.3) in order to obtain the particle spectrum in IC443. Having the proton spectrum, we calculate the hadronic $\gamma$-ray spectrum from this SNR (following the prescription of Kelner et al. 2006). The $\gamma$-ray spectrum from our model (Petruk et al. (2017)) is shown by the solid line on Fig. 4 (right plot). It fits the observations perfectly.

There are few conclusions from this figure. i) The spectrum of particles with the steadystate injection cannot explain the observed $\gamma$-ray spectrum. ii) The temporal evolution of the injection efficiency in the aftermath of the supernova considerably affects the $\gamma$-ray spectrum of SNR. iii) The break in the $\gamma$-ray spectrum of IC443 discovered by Ackermann et al. 2013 at the photon energies around $\sim 2 \mathrm{GeV}$ is a natural consequence of the smaller number of particles starting acceleration before the day 8000. iv) The early the particles were injected the more time they have for acceleration, the higher energies they may reach 


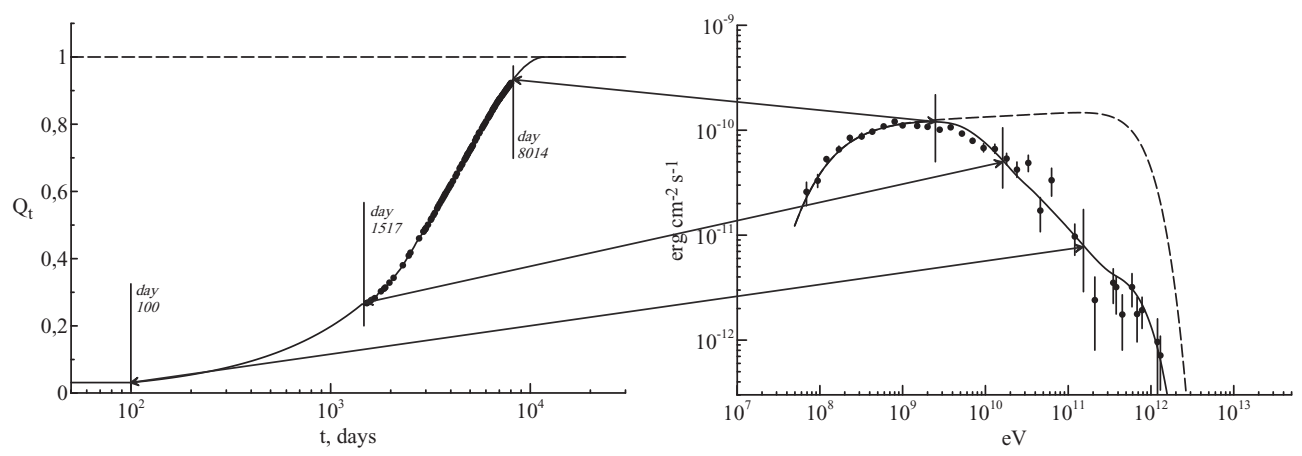

Figure 4. Evolution of $Q_{\mathrm{t}}$ (left) and the hadronic $\gamma$-ray spectrum of IC443 (right). The $\gamma$-ray observations of IC443 are reported by the Fermi (up to $10^{11} \mathrm{eV}$, Ackermann et al. 2013), MAGIC (Albert et al. 2007) and VERITAS (Acciari et al. 2009) collaborations. The solid line on the left plot shows $Q_{t}(t)$ and on the right plot represents the spectrum from our model. The dashed line shows the case of the steady-state injection. Adapted from Petruk et al. (2017).

and the more energetic $\gamma$-rays they are able to emit. Fig. 4 uncovers the correspondence between the proton injection time and the maximum energies of $\gamma$-rays which may be generated by these protons. It is quite impressive that hadronic $\mathrm{TeV} \gamma$-rays from SNR are emitted by protons injected just in the first few months after the supernova.

\section{On the visibility of SN1987A in hadronic gamma-rays}

We may use $Q_{\mathrm{t}}(t)$ derived from observations of SN1987A to estimate also the $\gamma$-ray visibility of this young SNR to the future experiment CTA.

If the acceleration time-scale in SN1987A is of the same order as in IC443, it is obvious that protons cannot be accelerated in SN1987A to the $\gamma$-ray emitting energies because of the difference in ages for these SNRs: time available for acceleration is small in SN1987A, $t_{\mathrm{sn} 87 \mathrm{a}} / t_{\mathrm{ic} 443} \simeq 0.01$. However, the right-hand part of the $\gamma$-ray spectrum (above $\sim 3 \mathrm{GeV}$ on Fig. 4) shifts toward the right with increase of $t / t_{1}$. Therefore, if the acceleration in SN1987A is faster (i.e. the smaller diffusion coefficient and the larger shock velocity) to the extent which provides

$$
\frac{t_{\mathrm{sn} 1987 \mathrm{a}}}{t_{\mathrm{ic} 443}} \frac{V_{\mathrm{sn} 1987 \mathrm{a}}^{2}}{V_{\mathrm{ic} 443}^{2}} \frac{D_{\text {ic } 443}}{D_{\mathrm{sn} 1987 \mathrm{a}}} \geqslant 1 .
$$

and the density of target protons in vicinity of SN1987A is $n_{\mathrm{pp}, \mathrm{sn} 87 \mathrm{a}} \geqslant 0.005 n_{\mathrm{pp}, \mathrm{ic} 443}$ then the hadronic $\gamma$-rays from SN1987A could be detectable by CTA (Fig. 5).

\section{Conclusions}

We use the test-particle solution of the time-dependent equation for the diffusive particle acceleration on the strong shocks in order to develop the method to reconstruct the temporal evolution of the injection efficiency (fraction of particles which begin acceleration). For this purpose, the observational data on the radio index of SN1987A were adopted.

It is shown that the spectrum of the highest-energy particles, in general, cannot be described by the steady-state equation with the constant injection. It is obligatory therefore to consider the non-stationary particle spectrum for models of the hadronic $\gamma$-ray emission from SNRs. Variation of the injection during the early times after the supernova explosion is critical for interpretation of the $\mathrm{TeV} \gamma$-ray spectra of SNRs. 


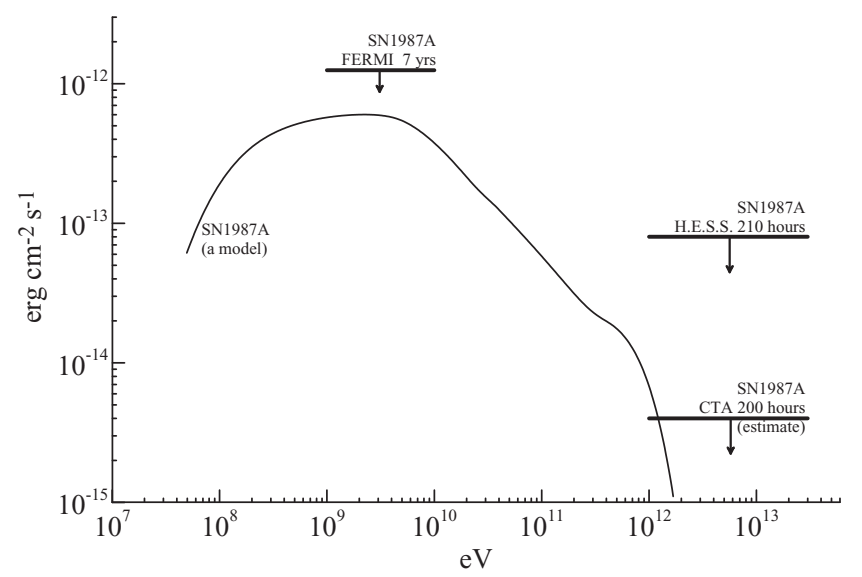

Figure 5. Eventual $\gamma$-ray spectrum of SN1987A. The upper limits on the $\gamma$-ray flux from SN1987A are from Ackermann et al. (2016) (FERMI), from H.E.S.S. Collaboration (2015) and the estimate for CTA is ours. Adapted from Petruk et al. (2017).

The radio observations of supernovae may be used to track the injection behavior. The main reason of the injection variation in the aftermath of the supernova is the rapid increase of the surface area of the forward shock.

In this approach, we have successfully fitted the $\mathrm{GeV}$-TeV $\gamma$-ray spectrum of IC443, using SN1987A as a proxy of the parent supernova. The break in the proton spectrum around $50-200 \mathrm{GeV}$ is a natural consequence of the time-dependent particle injection.

\section{Acknowledgements}

We thank G. Zanardo for the radio data on the spectral index evolution in SN1987A. We acknowledge support from the IAU and organizers to participate in the IAU Symposium 331. This paper was partially funded by the PRIN INAF 2014 grant 'Filling the gap between supernova explosions and their remnants through magnetohydrodynamic modeling and high performance computing'.

\section{References}

Acciari V. et al. 2009, ApJ, 698, L133

Ackermann M. et al. 2013, Science, 339, 807

Ackermann M. et al. 2016 A $\& A$ 586, 71

Albert J. et al. 2007, ApJ, 664, L87

Drury L. 1983, Rep. Prog. Phys., 46, 973

Forman M., Drury L. 1983, Proc. 18th ICRC, 2, 267

Jones F. 1990, ApJ, 361, 162

H. E. S. S. Collaboration 2015, Science, 347, 406

Kelner S., Aharonian F., Bugayov V. 2006, Phys. Rev. D, 74, 034018

Orlando S., Miceli M., Pumo M., Bocchino F. 2015, ApJ, 810, 168

Petruk O., Kopytko B. 2016, MNRAS, 462, 3104

Petruk O., Orlando S., Miceli M., Bocchino F. 2017, submitted

Skilling J. 1975, MNRAS, 172, 557

Zanardo G., Staveley-Smith L., Hg C.-Y. et al. 2013, ApJ, 767, 98 